\title{
Effect of Bundle Care on Deep Venous Thrombosis in Patients Received Renal Biopsy
}

\author{
Yingxue Zhong, Yuan He*, Ling Liu, Le Wen, Fanna Liu\#, Yu Chen\# \\ Nephrology Department, The First Affiliated Hospital of Jinan University, Guangzhou, China \\ Email: "81968920@qq.com, ${ }^{* 1139795771 @ q q . c o m ~}$
}

How to cite this paper: Zhong, Y.X., He, Y., Liu, L., Wen, L., Liu, F.N. and Chen, Y. (2021) Effect of Bundle Care on Deep Venous Thrombosis in Patients Received Renal Biopsy. International Journal of Clinical Medicine, 12, 183-189.

https://doi.org/10.4236/ijcm.2021.125017

Received: March 23, 2021

Accepted: May 17, 2021

Published: May 20, 2021

Copyright $\odot 2021$ by author(s) and Scientific Research Publishing Inc. This work is licensed under the Creative Commons Attribution International License (CC BY 4.0).

http://creativecommons.org/licenses/by/4.0/

\begin{abstract}
Objective: To explore the effect of bundle care on the incidence of venous thromboembolism (VTE) in patients who received renal biopsy, and provide reference for clinical care. Methods: 300 patients with nephrotic syndrome who received renal biopsy in our hospital from February 2018 to August 2020 were selected and randomly divided into the observation group and the control group, with 150 patients in each group. In the control group, patients were given routine care: informing the precautions before and after operation, observing the changes of vital signs and bleeding after operation, etc. In the observation group, patients were given bundle care intervention, including preoperative, intraoperative and postoperative routine care, ankle pump exercise, Intermittent Pneumatic Compression (IPC) treatment and psychological care. The incidence of lower-limb venous thrombosis was compared between the two groups. Results: The incidence of deep venous thrombosis in the observation group was lower than that in the control group $(\mathrm{P}<0.05)$, 1 case $(0.6 \%)$ in the observation group and 8 cases $(5.3 \%)$ in the control group; the peak velocity and mean velocity of lower-limb venous blood flow in the observation group were higher than those in the control group; the average length of stay in the observation group was less than that in the control group, and the satisfaction degree in the observation group was higher than that in the control group. The differences were statistically significant $(\mathrm{P}<$ 0.05). Conclusion: For patients who received renal biopsy, bundle care can help improve the peak velocity and mean velocity of venous blood flow, reduce the incidence of VTE, the average length of stay, and social costs, relieve their pain, and improve satisfaction degree of care; moreover, it plays an important role in reducing the incidence of deep venous thrombosis.
\end{abstract}

\section{Keywords}

Bundle Care, Renal Biopsy, VTE, Effect 


\section{Introduction}

"Bundle care" refers to a group of care interventions, each element of which is clinically proven to improve patient outcomes. Patient outcomes can be better improved under their joint implementation than single implementation. The application of "bundle care" can greatly increase the possibility of patients receiving care, so as to improve the overall quality of care [1]. Renal puncture biopsy is an invasive examination, in which a puncture needle is penetrated into the renal tissue under the positioning of B-ultrasound, and then a small amount of live tissue is taken for pathological examination. It is the gold standard for clinical diagnosis of renal diseases, especially glomerular diseases. It provides an important basis for the diagnosis, treatment, evaluated prognosis and diagnostic efficacy of renal diseases. At present, it has been widely used in clinic. VTE is one of the common complications after renal biopsy. The incidence of VTE reported in the literature is $3.63 \%-20.82 \%$. Therefore, in order to reduce the incidence of VTE in patients who received renal biopsy and relieve their pain, prevention is particularly important. In this study, 300 patients were selected to study the effect of bundle care on prevention of VTE after renal biopsy.

\section{Clinical Data}

General information 300 patients with nephrotic syndrome who received renal biopsy in our hospital from February 2018 to August 2020 were selected as subjects and they were randomly divided into control group and observation group, with 150 patients in each group. In the control group, there were 86 males and 64 females; the average age of patients was $41.8 \pm 1.8$ years old (from 18 to 68 years old); in the observation group, there were 96 males and 54 females; the average age of patients was $42.6 \pm 2.5$ years old (from 20 to 72 years old). For the differences in the general information between the two groups, there was no statistical significance $(P>0.05)$ (see Table 1$)$.

\section{Research Methods}

Control group Routine care of Nephrology Department the first diagnosis responsibility system was adopted for patients, and the nurse in charge was responsible

Table 1. Proportion of general information of patients (\%).

\begin{tabular}{ccccc}
\hline Subjects & Control group $(\mathrm{n}=150)$ & Observation group $(\mathrm{n}=150)$ & $\chi^{2}$ & $P$ \\
\hline Gender & & & 1.396 & 0.237 \\
Male & $86(57.3)$ & $96(64)$ & & \\
Female & $64(42.7)$ & $54(36)$ & & \\
Age/years old & & & 0.892 & 0.639 \\
$<40$ & $85(52.6)$ & $78(34.5)$ & & \\
$40-65$ & $56(42.1)$ & $64(55.2)$ & & \\
$>65$ & $9(5.3)$ & $8(10.3)$ & & \\
\hline
\end{tabular}


for the whole process of patients. The doctor in charge diagnosed and treated the patients according to the diagnosis and treatment routine, and the nurse in charge carried out routine care such as admission education, diet guidance, preoperative preparation and postoperative care according to the doctor's advice.

The observation group was given bundle care: on the basis of the control group, ankle pump exercise, IPC treatment and passive exercise were added, and the details are as follows:

\section{Care}

\subsection{Preoperative Preparation}

The nurse in charge guided the patients to cooperate with the doctor to do preoperative preparation well, including routine blood test, coagulation test, a set of virus tests, electrocardiogram, chest X-ray and B-ultrasound examination, so as to avoid the absolute contraindications in the operation. The changes of vital signs were monitored. The use of anticoagulant drugs such as vasoactive drugs was stopped three days before the operation to prevent postoperative bleeding. One day before the operation, the patients were trained to urinate in bed to avoid postoperative urinary retention. The intravenous indwelling needle was retained half an hour before the operation.

\subsection{Postoperative Care}

1) After renal puncture, the patients were sent back to the ward by flatcar; the situation of puncture site was checked, and an ECG monitor was used to monitor their vital signs, $\mathrm{QH}{ }^{*} 6$ times. The patients were guided to lie flat for 6 hours, and they can freely turn their heads, move both upper limbs, slightly move legs and feet under the condition of no relaxation and no exertion; the volume and color of urine were observed; bolsters were used to raise up both lower limbs by 20 - 30 degrees, making the distal end of lower limbs higher than the proximal end. The nurse in charge valued the patients' chief complaint, helped them to eat, and guided their amount of diet. 6 hours later, the nurse in charge removed the sandbag pressure at the puncture site, and helped the patients turn over.

2) Activities

a) Encourage the patients to breathe deeply. Studies have shown that $5 \mathrm{mi}$ nutes' active deep breathing exercise every 2 hours can help reduce the symptoms of Deep venous thrombosis (DVT) in the patients who received joint replacement, and also relieve the hypercoagulable state of blood, effectively lowering the risk of VTE [2]. The training of lip contraction and effective cough can increase diaphragm movement, reduce chest pressure and increase blood return [3], so as to prevent venous thrombosis.

b) Compress gastrocnemius muscle manually, and do passive massage from foot to thigh and from far to near, 30 minutes a time, 3 times a day [3]. 
c) Contract quadriceps femoris isometrically, straighten knee joint in supine position, and stretch thigh muscle for 5 seconds, relaxing for 2 seconds; or stretch for 10 seconds, relaxing for 10 seconds.

d) Ankle pump exercise. The ankle active "rotation" exercise had the strongest promoting effect on the femoral venous blood flow, which made the peak velocity of femoral venous blood flow increase by $69.3 \%$, mean velocity increasing by $69.1 \%[4]$.

i) Flexion-extension motion: the patients lied flat, stretched their lower limbs, relaxed their muscles and slowly hooked their feet, making their toes towards themselves to the maximum for 5 - 10 seconds; and then slowly pressed their toes down to keep them for 5 - 10 seconds to the maximum, and finally relaxed themselves. This was a set of motions, which could be repeated, at least 6 times a day.

ii) Rotation exercise: the patients, lying flat, stretching their lower limbs, and relaxing their muscles, centered on their ankles, and rotated their feet 360 degrees.

3) Intermittent pneumatic compression (IPC). At present, IPC is a common method to prevent DVT, which repeatedly pressurizes and depressurizes the affected limb and regularly inflates and deflates to prevent thrombosis [5] [6]. Before treatment: the medical staff explained to the patients the method and purpose of IPC, as well as the possible feelings or reactions of the body during the treatment. In addition, attention points should be emphasized with the patients during the treatment, and the comprehensive examination should be carried out to ensure the stable performance of the instrument. Firstly, the size of the pressure leg-sleeve was determined according to the circumference of the patient's lower limbs; and then during the treatment, the patient took the supine position, and the two lower limbs were wrapped in the pressure leg-sleeve, with the end of the inflation being set at $45 \mathrm{mmHg}$; the inflation was from the ankle to the thigh in turn, and then when the air bag was filled, the air was deflated. This process was cycled for a total of 40 minutes, once in the morning and afternoon every day.

4) Psychological care. The activity disorder caused by staying in bed brought great inconvenience to patients and affected their life and work. In addition, pain, infusion and other factors made patients nervous. Therefore, care staff should sympathize with and care for patients, guiding them to read books and listening to music, so as to distract their attention and reduce their pain. In the process of treatment, care staff should patiently explain the development process, treatment process and nursing points of the disease, so as to remove patients' doubts, enhance their confidence in overcoming the disease, and get their active cooperation.

5) Health education. As for diet, patients should eat light and digestible food with high calorie, high protein and vitamin and keep off cigarettes and alcohol. They were encouraged to drink water when their urine volume keeps a normal 
level; notably, for the patients with edema, the amount of water in and out should be accurately recorded, controlling the amount of drinking water (make ends meet). Their activities should be guided, and they should not eat the food or medicine of promoting blood circulation and removing blood stasis within 3 months, avoid bearing heavy load, and prevent the occurrence of cold and other infections.

\section{Observation Indexes}

1) Peak velocity and mean velocity of lower-limb venous blood flow and the incidence of VTE.

2) Length of stay. Record the time from admission to discharge between the two groups, and compare the average length of stay between the two groups.

3) Medical satisfaction survey. Through telephone follow-up, the medical satisfaction of discharged patients was investigated, including: very satisfied: 80 100 points; satisfied: $60-79$ points; dissatisfied: $<60$ points.

\section{Statistical Analysis}

SPSS23.0 was used for statistical analysis; t-test was used for comparison of measurement data, and $\chi^{2}$ test was used for comparison of count data; $\mathrm{P}<0.05$ means that the difference was statistically significant.

\section{Results}

\subsection{Incidence of Postoperative Complications in Two Groups}

Through $\chi^{2}$ test, the incidence of VTE in the observation group was significantly lower than that in the control group, and the peak velocity and mean velocity of venous blood flow were higher than those in the control group. The difference was statistically significant $(\mathrm{P}=0.0001)$ (see Table 2 ).

\subsection{Length of Stay}

The time from admission to discharge was recorded. Based on statistical analysis, the length of stay was observed, the length of stay in the observation group being significantly less than that of the control group $(\mathrm{P}<0.001)$ (see Table 3$)$.

Table 2. Incidence of VTE and peak velocity and mean velocity of lower-limb venous blood flow after operation $[(\mathrm{x}- \pm \mathrm{s}), \mathrm{cm} / \mathrm{s}]$.

\begin{tabular}{|c|c|c|c|c|}
\hline Group & Case & Incidence of VTE (\%) & Peak velocity & Mean velocity \\
\hline Observation group & 150 & 0.6 & $58.41 \pm 8.25$ & $31.14 \pm 5.23$ \\
\hline Control group & 150 & 5.3 & $43.33 \pm 8.05$ & $23.02 \pm 5.49$ \\
\hline $\mathrm{t}$ & - & 4.6 & 15.9 & 13.1 \\
\hline $\mathrm{p}$ & - & 0.03 & 0.0001 & 0.0001 \\
\hline
\end{tabular}


Table 3. Comparison of the length of stay between the two groups (days).

\begin{tabular}{cc}
\hline Group & The average length of stay \\
\hline Control group & $10.8 \pm 8.3$ \\
Observation group & $7.8 \pm 5.2$ \\
$\mathrm{t}$ & 3.75 \\
$\mathrm{P}$ & 0.0002 \\
\hline
\end{tabular}

Table 4. Comparison of medical satisfaction between the two groups (cases).

\begin{tabular}{ccccccc}
\hline Group & Case & Very satisfied & Satisfied & Dissatisfied & $\begin{array}{c}\text { Satisfaction } \\
(\%)\end{array}$ & $\begin{array}{c}\text { Total } \\
\text { satisfaction }\end{array}$ \\
\hline $\begin{array}{c}\text { Control } \\
\text { group }\end{array}$ & 150 & 94 & 20 & 36 & 76 & $114(76 \%)$ \\
$\begin{array}{c}\text { Observation } \\
\text { group }\end{array}$ & 150 & 120 & 18 & 7 & 94 & $138(92 \%)$ \\
$\chi^{2}$ & & & & & & 14.29 \\
$P$ & & & & & 0.0002 \\
\hline
\end{tabular}

\subsection{Medical Satisfaction Survey}

By $\chi^{2}$ test, the medical satisfaction rate of the observation group was significantly higher than that of the control group $(\mathrm{P}=0.0002)$ (see Table 4).

\section{Discussion}

VTE is a global health problem, seriously endangering human health. The occurrence of VTE is occult, and its clinical manifestations are not specific; $50 \%$ $80 \%$ of VTE have no clinical symptoms [7]. There are great differences in clinical symptoms and signs of VTE in different patients [8]. Patients who received renal puncture and biopsy are also the high-risk population of VTE. Once VTE is formed, and a long-term treatment is needed. Therefore, the following six aspects should be done: early screening, early prevention, early warning, early diagnosis, early treatment and early rehabilitation, which are the key points of VTE's prevention and treatment; and among them, the most important link is early warning [9].

This study discusses the application of bundle care to prevent the occurrence of VTE after renal biopsy; and based on Caprini assessment scale, this study effectively assesses the situation of patients by giving bundle care. The results show that the peak velocity and mean velocity of lower-limb venous blood flow in the observation group were higher than those in the control group, while the incidence of VTE was lower than that in the control group, and the medical satisfaction score was higher than that in the control group $(\mathrm{P}<0.05)$. It is confirmed that the application of bundle care intervention after renal biopsy plays an important role in preventing VTE and can help observe the disease changes or complications that may cause adverse prognosis in advance, which also reflects the comprehensive ability of care staff. Meanwhile, it improves the treatment 
level of departments, and reduces patients' pain, the average length of stay, and social costs, which improves patients' satisfaction. Therefore, the prevention of VTE after renal biopsy has important clinical and practical value, which is worthy of clinical application.

\section{Statement/Funding}

Novel coronavirus pneumonia patients with maintenance hemodialysis and medical care of integrated protection, Guangdong special fund for novel coronavirus pneumonia prevention and control research, Project No.: 2020kzdzx1033.

\section{Conflicts of Interest}

The authors declare no conflicts of interest regarding the publication of this paper.

\section{References}

[1] Resar, R., Pronovost, P., Haraden, C., et al. (2005) Using a Bundle Approach to Improve Ventilator Care Processes and Reduce Ventilator-Associated Pneumonia. Joint Commission Journal on Quality and Patient Safety, 31, 243-248. https://doi.org/10.1016/S1553-7250(05)31031-2

[2] Zhu, X. and Liu, H. (2020) The Influencing Factors and Intervention Measures of Deep Vein Thrombosis in Orthopedic Patients. Nursing Practice and Research, 17, 65-67.

[3] Shen, H. (2011) Prevention and Nursing of Deep Venous Thrombosis. Jilin Medical Journal, 14, 2910-2911.

[4] Wen, Y. and Deng, Z. (2012) Nephrotic Syndrome and Thrombosis Forecasting Factor Analysis and Nursing Countermeasures. Medical Journal of West China, 2, 412-413.

[5] Xu, X. (2016) Intervention Effect of Intermittent Pneumatic Compression Therapy on the Occurrence of Venous Thromboembolism in Severe Patients. Journal of China Prescription Drug, 14, 111-112.

[6] Huang, X., Su, Z., Yang, R., et al. (2017) Clinical Study of Preyent Deep Vein Thrombosis by Intermittent Pneumatic Compression Devices for the Patints Accepted Urology Lithotomy Position Operation in Preoperative Early Stage. China Medical Device Information, 23, 1-2.

[7] Qin, L. and Lu, W. (2013) The Progress of Diagnosis and Treatment of Venous Thromboembolism. Chinese Journal of Tuberculosis and Respiratory Diseases, 36, 372-374.

[8] Wen, S. and Liu, J. (2010) Epidemiology of Venous Thromboembolism. Chinese Journal of Geriatric Heart Brain and Vessel Diseases, 12, 961-963.

[9] Xia, Y., Du, W. and Peng, L. (2017) Application of VTE Nursing Strategy System Based on Autar Score in Patients with Pelvic and Lower-Limb Fractures. Today Nurse (Mid-Periodical), 24, 34-36. 